\title{
Microscopic and spectral dosimetry using GaF-chromic films and surface electron microscopy
}

F. Van den Heuvel*, G. De Kerf*, J.W. Seo ${ }^{\dagger}$, E. Van Limbergen*, E. Couteau ${ }^{\ddagger}$, S. Nuyts*, and J.P. Locquet ${ }^{\ddagger}$

$\left(^{*}\right)$ Dept of Experimental Radiotherapy, $\left({ }^{\dagger}\right)$ Dept. Metallurgy and Material Engineering, $\left(^{\ddagger}\right)$ Solid State Physics and Magnetism Section University of Leuven, Leuven, Belgium

Abstract

Purpose/Objective(s): The use of high-Z nano-particles (NP) increases the dose in the nearby volume by absorbing more photons or kev energy. The resulting electrons deposit the dose locally. Additionally, Auger electrons are generated. These very low energy electrons can contribute to dose in a high LET mode nation with tumor specific agents such a dose deposition mechanism could provide an efficient methodology to increase dose to microscopic disease in addition to dose normally delivered to the tumor.To quantify dose enhancement and spectral changes in the vicinity of these particles we propose the use of GaFchromic film which can be analyzed using scanning electron mcroscopes (SEM) to yield microscopic insight in the processes 政 Ims under these conditions.

\section{Methods and Materials}

\subsection{Irradiating samples}

In this work we applied two different experimental setups in or-

1.2 Experimental setup — diagnostic energies

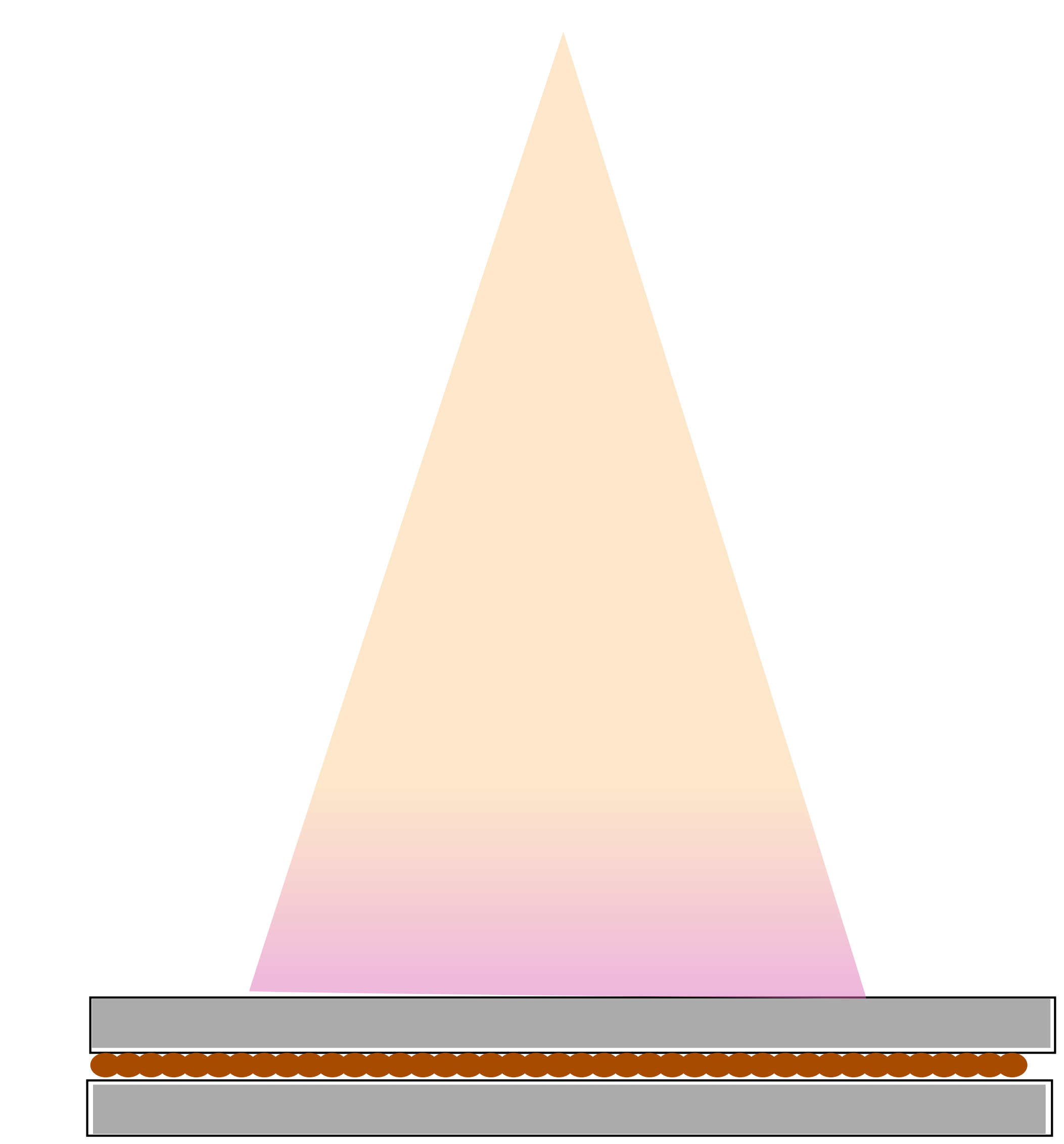

Figure 1: Experimental setup

Figure 1 shows the experimental setup. Pure gold nanoparticles of size $30 \mathrm{~nm}$ are layered on the top of a GaFChromic film was stripped of its protective layers so that only the active part was used. Another film is positioned on top of this film. Films without Nano-particles where irradiated with photons from a standard clinical X-ray (Acuity, Varian Medical Systems, Milpitas, CA) machine set to a nominal energy of $40 \mathrm{kVp}$. The output was calibrated to deliver 10cGy in water. The irradiation was performed on fims with and win in GN. Th. Furhermore the GNP
area.

1.3 Experimental setup - Megavoltage energies Films were embedded in a stack of solid water (RW-3) at a depth of $5 \mathrm{~cm}$ with a backscatter thickness of $10 \mathrm{~cm}$. Doses of 1 and 2 Gy were delivered to the different films. This was done to ogy.

\subsection{Measurements}

The samples were characterized by means of the scanning Nanolab operating at 2kV and 53pA. As the GaF Chromic films are not conductive, the surface is coated prior to SEM observations with a thin layer of Au in a sputter coater, which uses an Ar-plasma on a gold target (deposition time of about 1 min.). heating $\left(<50^{\circ} \mathrm{C}\right)$ occurs during the deposition.

\subsection{Analysis}

The diagnostic films were reduced to a skeleton image using thresholding and a mathematical morphology technique. For in a histogram Using a polynomial approximation of the CSDA path length for electrons, we converted the energy spectrum of the depositing electrons to a pathlength histogram to compare with the measured histogram.

\subsection{Radiobiological effect}

To estimate the relative effect of the change in spectrum and the increase in the contribution by Auger-electrons we used a fast monte carlo model of biological damage as proposed by Semeresults as track code monte carlo codes as proposed by Nikjoo et al.. The approach used here determines the amount of different damage to a DNA-molecule from direct ionization as well as through the generation of radicals. It then produces a yield single strand breaks to complex clustered double strand breaks for interacting electrons of a given energy $(E)$. The methodology used to incorporate this information in our calculations can be found in recent article published by our group[3]

\section{Results}

2.1 Biological effect

Figure 2 shows the yield per cell and per Gy of single strand breaks (SSB) and double strand breaks (DSB) as a function of energy. This for the three volumes under consideraion. The target volume containing GNP reflects the atomic structure in the calculated yields. One can easily identify the energy. The addition generates increases in DSB and lowers the contributions of SSB. However, the added tail of high energy electrons mainly affects the DNA through SSB's, It is only for very low energies $(10-20 \mathrm{keV})$ that the addition of GNP changes the ratio of SSB to DSB to give the dose deposition a more high LET character. For deeper lying structures there is no data avallable for $10 \mathrm{keV}$ as the photons do not penetrate deep enough to generate a meaningful contribution.

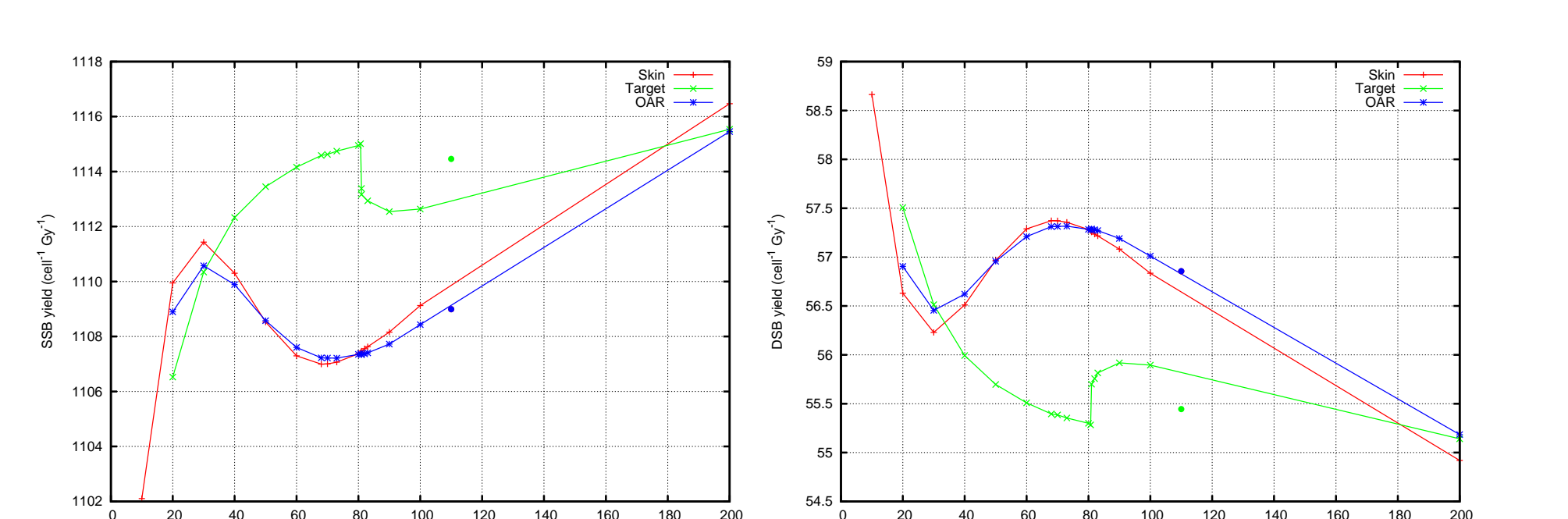

Figure 2: Plots for generation of single strand breaks (left) and double strand breaks (right) as a function of energy for a given goes down the DSB's go up. In the target volume the atomic structure and resonant levels generating auger electrons are visible but have a limited impact on the overall result with respect to the deposition being of a high or low LET nature.

\subsection{Experimental results}

Figure 3 shows the results from a monte carlo simulation where the preponderance of auger-events was tallied. The efficiency Figure 4 we clearly see that dose enhancement takes place. As the increase is uniform on the top and the bottom of the film in contact with the GNPs we presume that the enhancement is purely due to increased photo-electric interactions. The paucity of auger-events in the bottom part of the GNP contacted film corroborates the low yield of these events from the simulation. Further simulations (data not shown) suggest a higher count of auger-events but they did not increase the relative number of double strand breaks. However, this might

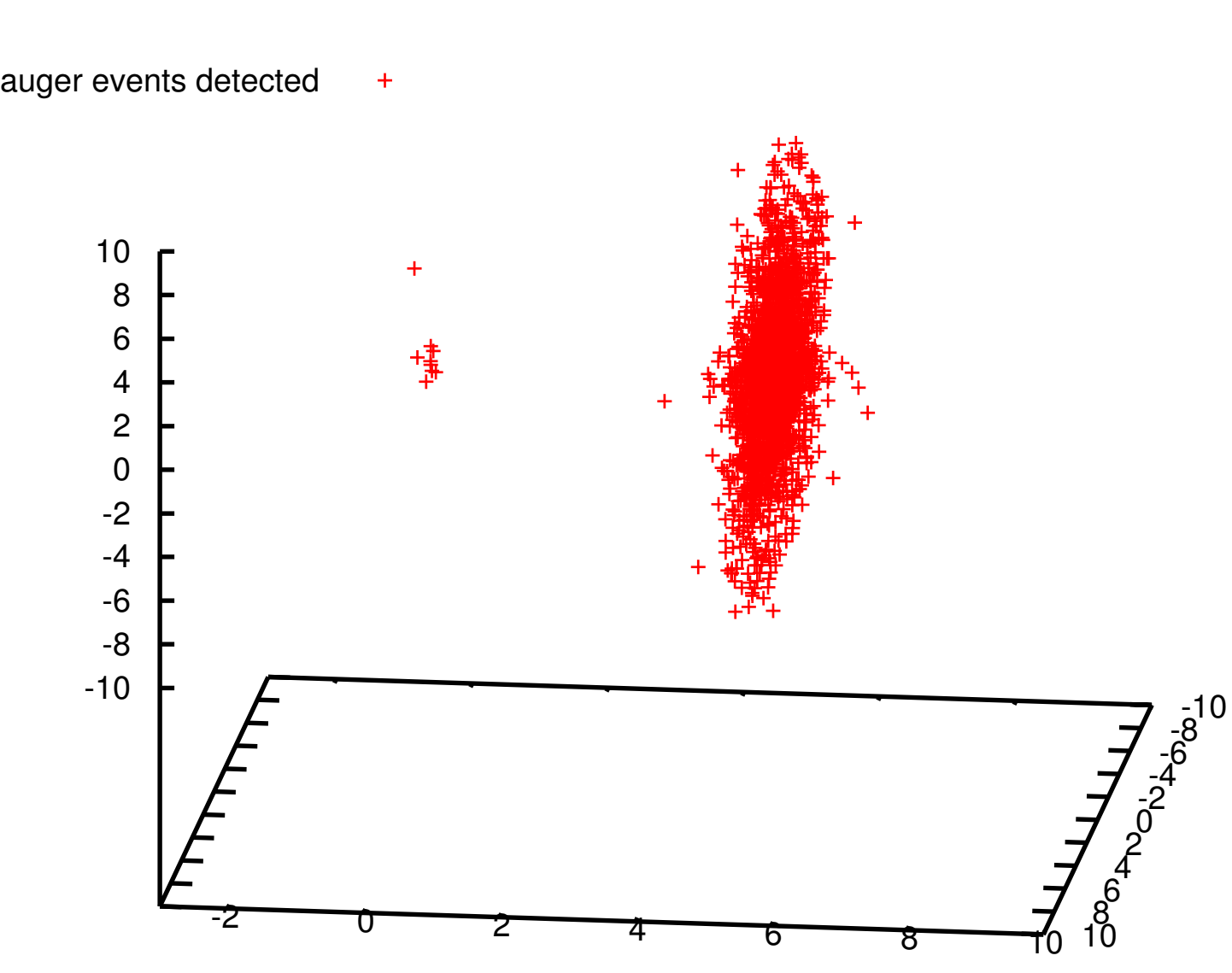

Figure 3: Determination of the position of auger-events genwith a layer of $1 \mathrm{p}$-ray beam impinging on a water phantom depth. The yield of Auger-events is about 1\%

\subsection{Diagnostic Beams}

Figure 4 shows the histogram for a 20cGy dose deposition. The conversion to a tracklength histogram is compared to a CSDA histogram of a Monte Carlo simulation. Although the ist. Most importantly a large peak in the Monte Carlo data is not present in the measurement data. Two possibilities are hy pothesized: 1) A large background of unrelated short tracks even lower energies. 2) The Monte Carlo simulation is not able to track electrons with energies lower than 10keV. Stopped electrons are counted as soon as the threshold is reached.

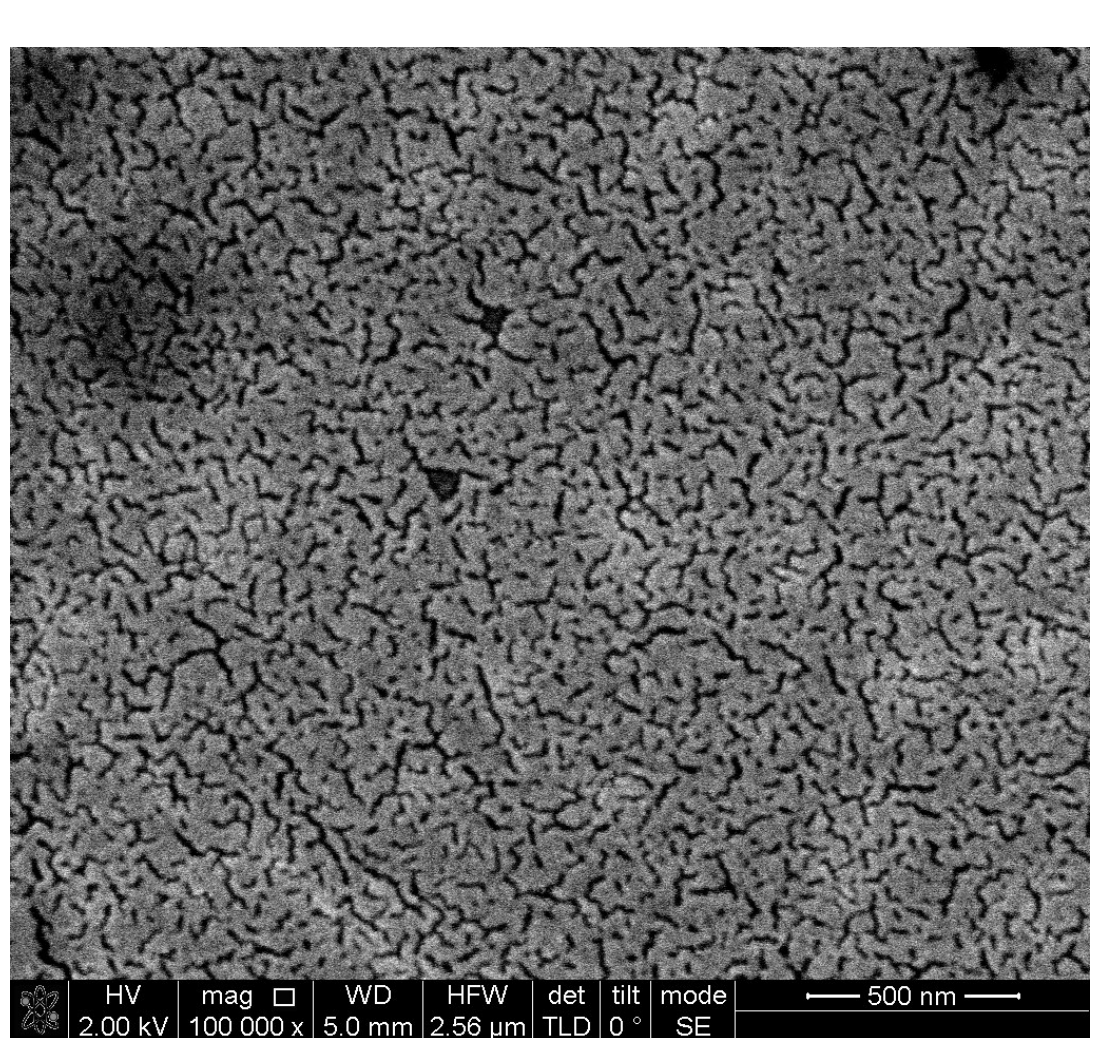$$
\text { (a) 200Gy@ 40keV }
$$
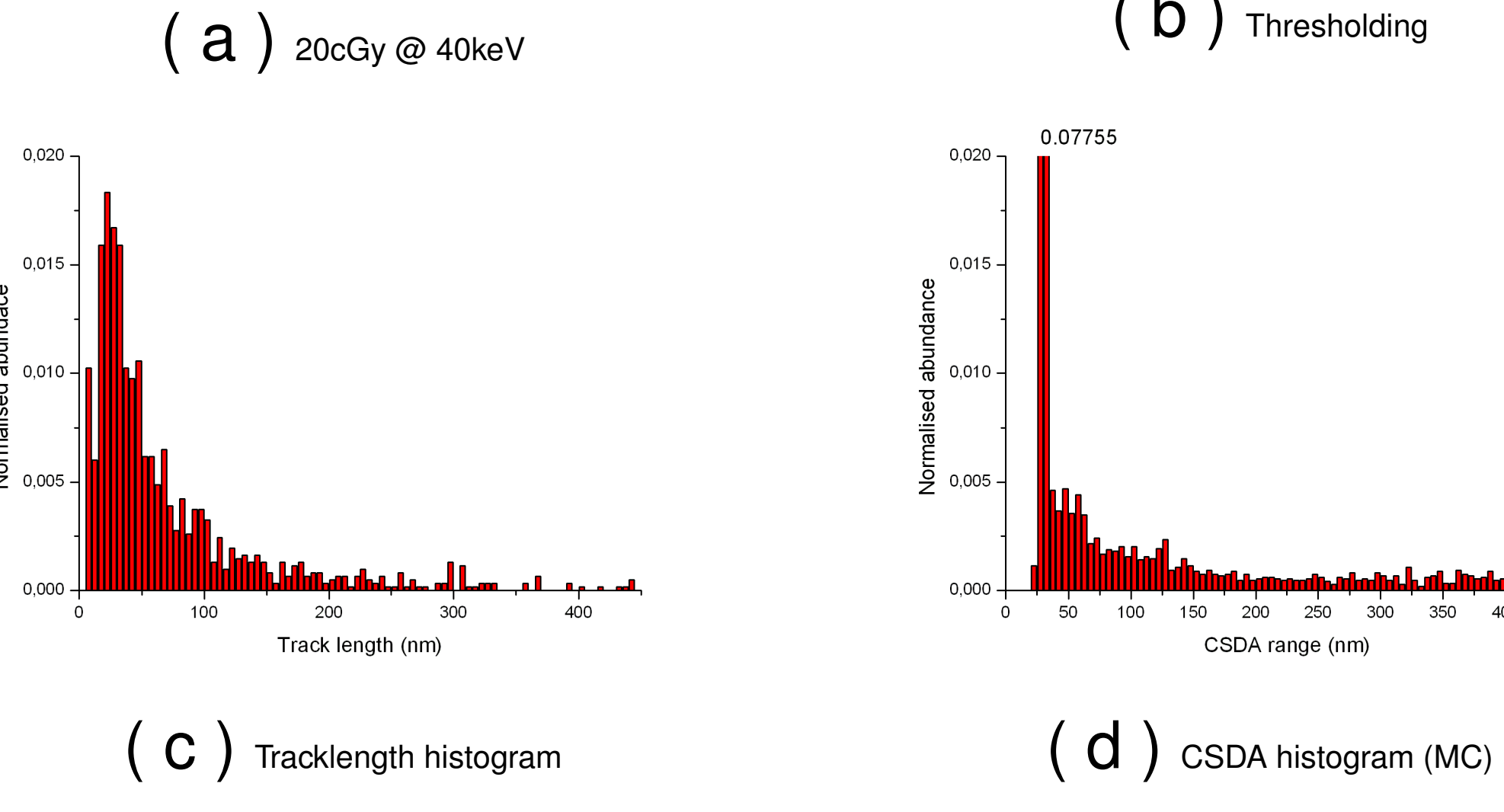

Figure 4: Scanning electron microscopic image of a film irradiated at 20cGy using $40 \mathrm{kVp}$ X-rays (right top), The film is skeletonized and palnhengths are presented in a histogram. The image from the bottom right is the Monte carlo simulation per-
formed at this energy in the presence of GNP's

\subsection{Megavoltage beams}

Due to the low flux of the diagnostic machine it is difficult to obtain higher doses. We therefore resorted to a linear accelerator to better analyze the response of the films. In figure ?? the images clearly show a difference between the reference image and the 1 and 2Gy images. The reference film also shows many trast in the imare thinner than the counterparts. Also the conused in the diagnostic images does not work as too many features detected in the image are not related to the radiation. $A$ multiresolution approach is likely more suited for this purpose but is still a work in progress. Manual selection of the thicker tracks in the image does show a doubling in total length (e.g. to-
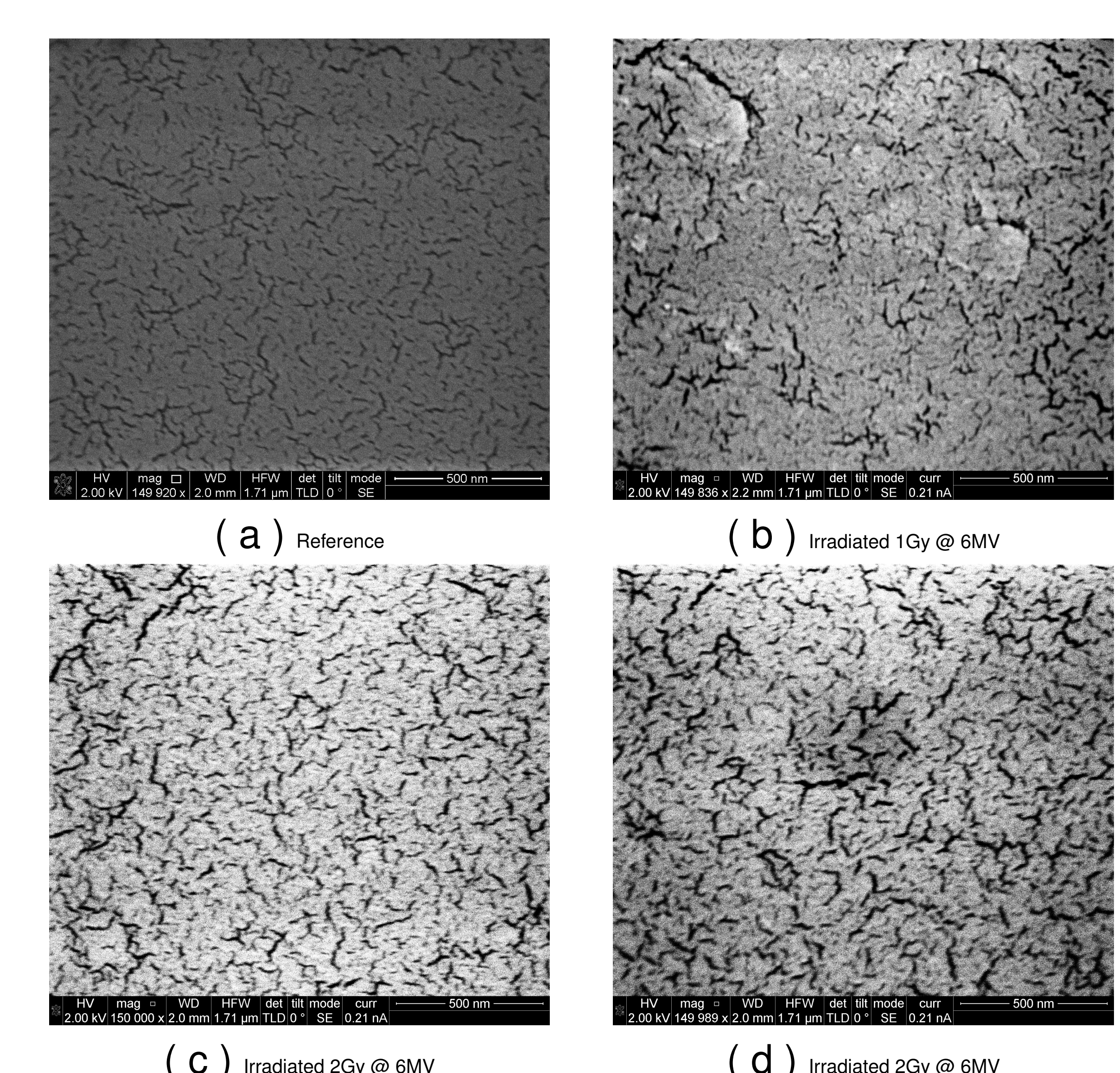

(d)

Figure 5: Scanning electron microscopic images of an unirradiated reference film (left top), a film irradiated at 1 Gy using $6 M V X$-rays (right top). The two bottom films both have received
$2 G y$

\section{Conclusions}

We have shown that Auger electrons do contribute to the dose enhancement. The contribution of these events is about $1 \%$ the interactions, which could be fairly low. At higher energles we expect the contribution to go up as more lower shell electron way to quantify the interactions at microscopic leve Althood we have candidates for auger-events it is a stretch to say tha we can link the candidates definitely to these events. In order to obtain certainty experiments at higher energies need to be performed where we can match the change in preponderance of events to the number of candidates seen.

References

[1] L. S. Waters, G. W. McKinney, J. W. Durkee, M. L. Fensin, J. S. Hendricks, M. R. James, R. C. Johns, and D. B. Pelowitz. The MCNPX Monte Carlo radiation transport code. In Albrow, M and Raja, R,

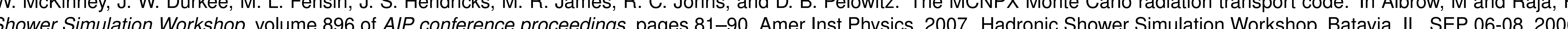
[2] V. Semenenko and R. Stewart. Fast Monte Carlo simulation of DNA damage formed by electrons and light ions. Phys Med Biol, 51(7):1693-1706, APR 72006

[3] F. Van den Heuvel, J.-P. Locquet, and S. Nuyts. Beam energy considerations for gold nano-particle enhanced radiation treatment. Physics in Medicine and Biology, 55(16):4509, 2010. 